\title{
Instability of a high-speed submerged elastic jet
}

\author{
By J. M. RALLISON AND E. J. HINCH \\ Department of Applied Mathematics and Theoretical Physics, The University of Cambridge, \\ Silver Street, Cambridge CB3 9EW, UK
}

(Received 26 August 1994)

The linearized inertial instability of the parallel shear flow of a viscoelastic liquid is considered. An elastic Rayleigh equation is derived, for high Reynolds numbers and high Weissenberg numbers, and for a viscoelastic liquid whose first normal stress dominates other stresses. The equation is used to investigate the stability of a submerged jet, that may be planar or axisymmetric, having a parabolic velocity profile. The sinuous mode is found to be fully stabilized by sufficiently large elasticity. The varicose mode in the planar case is partially stabilized, being unstable only at longer wavelengths and with a reduced growth rate. An axisymmetric jet, which is stable to varicose perturbations at zero elasticity, is found to be unstable to shortwave disturbances for small non-zero elasticity. This novel instability involves elastic waves in the shear. It is also present in other modes but does not have the fastest growth rate.

\section{Introduction}

In a recent paper Azaiez \& Homsy (1994) studied the stabilizing effect of elastic normal stresses on the inertial instability of a free shear layer, the Kelvin-Helmholtz instability. In order to isolate the inertial instability, they considered high Reynolds numbers. They found that there was a significant stabilization only for non-Newtonian fluids with large normal stresses, and among these only for the Oldroyd-B fluid at high Weissenberg numbers. For the free shear layer, Azaiez \& Homsy found the growth rate was reduced by the elastic stresses and the unstable wavelengths became long.

In this paper we consider another inertial instability, that of a submerged jet having a parabolic velocity profile at high Reynolds number. We selected this geometry because there are some problems in establishing consistently the undisturbed free shear flow, whereas our parabolic profile could be established in a pipe feeding the jet. Following Azaiez \& Homsy we consider an Oldroyd-B fluid at high Weissenberg number. Additionally we will consider only the temporal instability of a spatially infinite unidirectional base flow. Our geometry is either planar or axisymmetric.

There are some practical problems in applying our analysis to a real jet. A real jet evolves slowly downstream, and for such a jet an analysis of the spatial instability may be more appropriate. Also there may be a die-swell, particularly at low speeds, which changes significantly the parabolic velocity profile in a the nozzle. We ignore these practical problems in this paper.

The inertial instability of a submerged jet in a Newtonian fluid has been considered by many, motivated in particular by the noise generated by aircraft engines. Various velocity profiles have been studied in two dimensions and in axisymmetric flows. 
For example Bickley (1937) and Drazin \& Howard (1966) considered the inviscid two-dimensional flow with a $\operatorname{sech}^{2} y$ profile, and Batchelor \& Gill (1962) considered inviscid axisymmetric jets with top-hat and parabolic velocity profiles. In general a sinuous mode is found to be more unstable than a varicose mode. In fact Batchelor $\&$ Gill found no unstable varicose mode for an axisymmetric submerged jet with a parabolic profile.

\section{An elastic Rayleigh equation}

Consider the flow of an Oldroyd-B fluid governed by

$$
\begin{gathered}
\nabla \cdot \boldsymbol{u}=0 \\
\rho \frac{\mathrm{D} \boldsymbol{u}}{\mathrm{D} t}=-\nabla p+\mu \nabla^{2} \boldsymbol{u}+G \nabla \cdot \boldsymbol{A}+\boldsymbol{f}, \\
\frac{\mathrm{D} \boldsymbol{A}}{\mathrm{D} t}=\boldsymbol{A} \cdot \boldsymbol{\nabla} \boldsymbol{u}+\nabla \boldsymbol{u}^{T} \cdot \boldsymbol{A}-\frac{1}{\tau}(\boldsymbol{A}-\boldsymbol{I}),
\end{gathered}
$$

where $G$ is the elasticity modulus and $\tau$ the relaxation rate associated with the polymer stretch $\boldsymbol{A}$.

We take as the unperturbed base state a general unidirectional flow

$$
\boldsymbol{u}=(U(y, z), 0,0),
$$

with a cross-sectional length scale $b$. The associated polymer stretch in the base state is

$$
\boldsymbol{A}=\left(\begin{array}{ccc}
1+2 \tau^{2}(\partial U / \partial y)^{2}+2 \tau^{2}(\partial U / \partial z)^{2} & \tau \partial U / \partial y & \tau \partial U / \partial z \\
\tau \partial U / \partial y & 1 & 0 \\
\tau \partial U / \partial z & 0 & 1
\end{array}\right)
$$

A body force $f$ is needed to maintain this base state.

We make two approximations. First that the Reynolds number $\rho U b /(\mu+G \tau)$ is large. This enables us to concentrate on the inertial instability, and to ignore the diffusion of momentum. Second that the Weissenberg number $U \tau / b$ is large. This provides the large normal stresses and allows us to ignore stress relaxation during the instability. The elastic stress in the basic state is then the normal stress $G A_{11}$. Using lower case letters to denote perturbed quantities, the equations governing the linear instability then become

$$
\begin{aligned}
\frac{\partial u}{\partial x}+\frac{\partial v}{\partial y}+\frac{\partial w}{\partial z} & =0 \\
\rho\left[\left(\frac{\partial}{\partial t}+U \frac{\partial}{\partial x}\right) u+v \frac{\partial U}{\partial y}+w \frac{\partial U}{\partial z}\right] & =-\frac{\partial p}{\partial x}+G\left[\frac{\partial a_{11}}{\partial x}+\frac{\partial a_{12}}{\partial y}+\frac{\partial a_{13}}{\partial z}\right], \\
\rho\left(\frac{\partial}{\partial t}+U \frac{\partial}{\partial x}\right) v & =-\frac{\partial p}{\partial y}+G \frac{\partial a_{12}}{\partial x} \\
\rho\left(\frac{\partial}{\partial t}+U \frac{\partial}{\partial x}\right) w & =-\frac{\partial p}{\partial z}+G \frac{\partial a_{13}}{\partial x}, \\
\left(\frac{\partial}{\partial t}+U \frac{\partial}{\partial x}\right) a_{11}+v \frac{\partial A_{11}}{\partial y}+w \frac{\partial A_{11}}{\partial z} & =2 A_{11} \frac{\partial u}{\partial x}+2 a_{12} \frac{\partial U}{\partial y}+2 a_{13} \frac{\partial U}{\partial z} \\
\left(\frac{\partial}{\partial t}+U \frac{\partial}{\partial x}\right) a_{12} & =A_{11} \frac{\partial v}{\partial x}
\end{aligned}
$$




$$
\left(\frac{\partial}{\partial t}+U \frac{\partial}{\partial x}\right) a_{13}=A_{11} \frac{\partial w}{\partial x} \text {. }
$$

It should be noted that the high-Reynolds-number approximation of ignoring the small tangential viscous stresses has the effect of suppressing some modes of instability such as the co-extrusion instability discussed by Hinch, Harris \& Rallison (1992). Furthermore, although the elastic tangential stresses in the base state $A_{12}, A_{13}$ are smaller than the basic normal stress $A_{11}$ by a factor $b / U \tau$ and so are negligible, the perturbation tangential stresses $a_{12}, a_{13}$ are forced by $A_{11}$ and so must be retained.

We consider a temporal instability where all perturbation quantities are functions of $y$ and $z$ and are proportional to $\mathrm{e}^{\theta}$ with $\theta=\mathrm{i} \alpha(x-c t)$. The growth rate is thus $\sigma=\alpha c_{i}$.

Solving for the stress perturbations we find

$$
\begin{gathered}
a_{12}=A_{11} v /(U-c), \quad a_{13}=A_{11} w /(U-c), \\
a_{11}=\frac{1}{\mathrm{i} \alpha(U-c)}\left[2 A_{11} \mathrm{i} \alpha u+\left(\frac{2 A_{11}}{U-c} \frac{\partial U}{\partial y}-\frac{\partial A_{11}}{\partial y}\right) v+\left(\frac{2 A_{11}}{U-c} \frac{\partial U}{\partial z}-\frac{\partial A_{11}}{\partial z}\right) w\right] .
\end{gathered}
$$

Substituting these into the momentum equation, we obtain

$$
\begin{aligned}
\left(\rho-\frac{G A_{11}}{(U-c)^{2}}\right)\left(\mathrm{i} \alpha(U-c) u+v \frac{\partial U}{\partial y}+w \frac{\partial U}{\partial z}\right) & =-\mathrm{i} \alpha p, \\
\left(\rho-\frac{G A_{11}}{(U-c)^{2}}\right) \mathrm{i} \alpha(U-c) v & =-\frac{\partial p}{\partial y}, \\
\left(\rho-\frac{G A_{11}}{(U-c)^{2}}\right) \mathrm{i} \alpha(U-c) w & =-\frac{\partial p}{\partial z} .
\end{aligned}
$$

A physically more revealing form of these expressions may be obtained by making use of the streamline displacements in the cross-section. (For a planar flow these displacements are closely related to the streamfunction.) With small streamline displacements $(\eta, \zeta)$ of the basic flow, the perturbation flow in the cross-section is given by

$$
v=\mathrm{i} \alpha(U-c) \eta \quad \text { and } \quad w=\mathrm{i} \alpha(U-c) \zeta .
$$

Mass conservation then gives the perturbation flow in the $x$-direction

$$
u=-\eta \frac{\partial U}{\partial y}-\zeta \frac{\partial U}{\partial z}-(U-c)\left(\frac{\partial \eta}{\partial y}+\frac{\partial \zeta}{\partial z}\right) .
$$

The first two terms represent the velocity maintaining its initial value as the streamlines are displaced, while the last terms represent the acceleration of the flow necessary as the streamlines crowd together, $\partial \eta / \partial y+\partial \zeta / \partial z<0$.

With this transformation, the perturbation stresses simplify to

$$
a_{12}=\mathrm{i} \alpha \eta A_{11} \text { and } a_{13}=\mathrm{i} \alpha \zeta A_{11},
$$

corresponding to a tilting of the streamlines that carry the basic stress, and

$$
a_{11}=-2 A_{11}\left(\frac{\partial \eta}{\partial y}+\frac{\partial \zeta}{\partial z}\right)-\left(\eta \frac{\partial A_{11}}{\partial y}+\zeta \frac{\partial A_{11}}{\partial z}\right) .
$$

In the last expression, the final pair of terms represents the initial basic stress being maintained on the displaced streamlines, while the first pair represents an enhancement of the stress generated by compressing the streamlines. 
The momentum equations now become

$$
\begin{aligned}
{\left[\rho(U-c)^{2}-G A_{11}\right]\left(\frac{\partial \eta}{\partial y}+\frac{\partial \zeta}{\partial z}\right) } & =p \\
{\left[\rho(U-c)^{2}-G A_{11}\right] \alpha^{2} \eta } & =\frac{\partial p}{\partial y}, \\
{\left[\rho(U-c)^{2}-G A_{11}\right] \alpha^{2} \zeta } & =\frac{\partial p}{\partial z} .
\end{aligned}
$$

The first equation expresses the balance between three effects: an acceleration in the $x$-direction when the streamlines crowd together, the extra elastic stress in the $x$-direction also caused by the streamlines crowding, and the pressure gradient in the $x$-direction. The equations in the $y z$-cross-section express a balance between a centrifugal acceleration around the curved streamlines, an elastic hoop stress around the curved streamlines, and the pressure gradient in the cross-section.

The streamline displacements $(\eta, \zeta)$ and the pressure $p$ must be continuous everywhere (e.g. at the edge of the jet). These requirements provide appropriate boundary conditions for the momentum equation.

It should be noted that, although our derivation above is for an Oldroyd-B fluid, these equations apply to a wider class of elastic liquids. We have assumed only that the normal stress $A_{11}$ dominates the base state, and that the perturbation stresses are the base state stresses co-deformed by the perturbation flow and with no stress relaxation on the time scale of the instability. Thus any constitutive equation involving an affine time derivative will give the same result at high Weissenberg numbers.

For a planar flow with streamfunction $\psi(x, y)=\mathrm{e}^{\theta} \phi(y)$ the streamline displacements are

$$
\zeta=0 \text { and } \eta=-\phi /(U-c)
$$

giving the elastic Rayleigh equation derived by Azaiez \& Homsy:

$$
\frac{\mathrm{d}}{\mathrm{d} y}\left[\left(\rho(U-c)^{2}-G A_{11}\right) \frac{\mathrm{d}}{\mathrm{d} y} \frac{\phi}{U-c}\right]=\left(\rho(U-c)^{2}-G A_{11}\right) \frac{\alpha^{2} \phi}{U-c} .
$$

More generally we may eliminate $\eta$ and $\zeta$ to form a single scalar equation for the pressure $p(y, z)$

$$
\left[\rho(U-c)^{2}-G A_{11}\right] \nabla_{\perp} \cdot \frac{1}{\rho(U-c)^{2}-G A_{11}} \nabla_{\perp} p=0
$$

where $\nabla_{\perp}$ operates in the $y z$-cross-section. This self-adjoint form yields a standard semi circle theorem (Howard 1961) which says that the complex wave speed $c$ must lie within a circle centred on $\frac{1}{2}\left(U_{\max }+U_{\min }\right)$ having radius $\frac{1}{2}\left(U_{\max }-U_{\min }\right)$. The effect of the elasticity $G A_{11}$ term is to reduce the radius of the circle slightly below this estimate. In practice in preference to this single equation for $p$ we prefer to use the separate equations above for $\eta, \zeta$, and $p$ since they make the associated boundary conditions more natural.

The presence of elasticity in the flow introduces the possibility of propagation of small-scale disturbances as elastic waves on the tensioned streamlines at a speed $c_{s}=\left(G A_{11} / \rho\right)^{1 / 2}$ relative to the flow. This speed of course exceeds the shear wave propagation speed $(G / \rho)^{1 / 2}$ appropriate for the unstressed state and for propagation of disturbances perpendicular to the streamlines.

The elastic form of the Rayleigh equation contains a new dimensionless group $E$ 
giving the ratio of elastic and inertial terms as

$$
E=\frac{G A_{11}}{\rho U^{2}}=\frac{G \tau^{2}}{\rho b^{2}}
$$

since $A_{11} \approx U^{2} \tau^{2} / b^{2}$, where $b$ is the length scale of the variations of $U(y, z)$. This elasticity number may alternatively be viewed as the square of an elastic Mach number $U / c_{s}$ appropriate for the stressed basic state. The remarkable independence of this number from the magnitude of the velocity arises because both the Reynolds and elastic stresses increase quadratically with the velocity.

It is important for the self-consistency of our analysis that the basic flow should not be modified by viscosity or elasticity on the time scale of the instability which is being studied, i.e. on the time scale $b / U$. Later numerical computations show that this estimate is correct for the fastest growing mode within a factor of order unity. Vorticity diffuses on the time scale $\rho b^{2} / \mu$ which is long compared with $b / U$ provided that the Reynolds number $\rho U b / \mu$ is large, as assumed. The elastic stress relaxes on the time scale $\tau$; again long if the Weissenberg number $U \tau / b$ is large. Finally the time for shear waves to propagate across the jet at the speed $(G / \rho)^{1 / 2}$ may be written as $(U \tau / b)^{1 / 2} \times(\rho U b / \mu)^{1 / 2} \times(\mu / G \tau)^{1 / 2} \times b / U$, which is also long if the Weissenberg and Reynolds numbers are large and $\mu / G \tau$ is not small.

\section{Two-dimensional jets}

We consider a base flow of a submerged jet of width $b$ and maximum velocity $U_{0}$ and with a parabolic profile

$$
U=\left\{\begin{array}{ll}
U_{0}\left(1-y^{2} / b^{2}\right) & \text { in }-b<y<b \\
0 & \text { otherwise }
\end{array} .\right.
$$

While it is simplest to imagine that the fluids inside and outside the jet are identical, within the approximation of high Reynolds and Weissenberg numbers any miscible pair of fluids having the same density could be involved. The linear stability theory does not 'see' the rheology of the fluid outside the jet. The associated base stress for the Oldroyd-B fluid is

$$
G A_{11}=\left\{\begin{array}{ll}
8 G \tau^{2} U_{0}^{2} y^{2} / b^{4} & \text { in }-b<y<b \\
0 & \text { otherwise }
\end{array} .\right.
$$

We non-dimensionalize the problem using the velocity $U_{0}$ and the length $b$. This results in one non-dimensional group measuring the strength of the elasticity $E=G \tau^{2} / \rho b^{2}$. The elastic Rayleigh equation then becomes

$$
\begin{gathered}
{\left[\left(1-y^{2}-c\right)^{2}-8 E y^{2}\right] \frac{\mathrm{d} \eta}{\mathrm{d} y}=p,} \\
{\left[\left(1-y^{2}-c\right)^{2}-8 E y^{2}\right] \alpha^{2} \eta=\frac{\mathrm{d} p}{\mathrm{~d} y},}
\end{gathered}
$$

within the jet $-1<y<1$, and outside the jet the same equations apply except that the square bracket is replaced by $c^{2}$. The boundary conditions at the edge of the jet are that the streamline displacement $\eta$ and the pressure $p$ are continuous.

We will examine the stability both of a sinuous mode which has streamline displacements symmetric about the centreline $y=0$ and of a varicose mode which has 


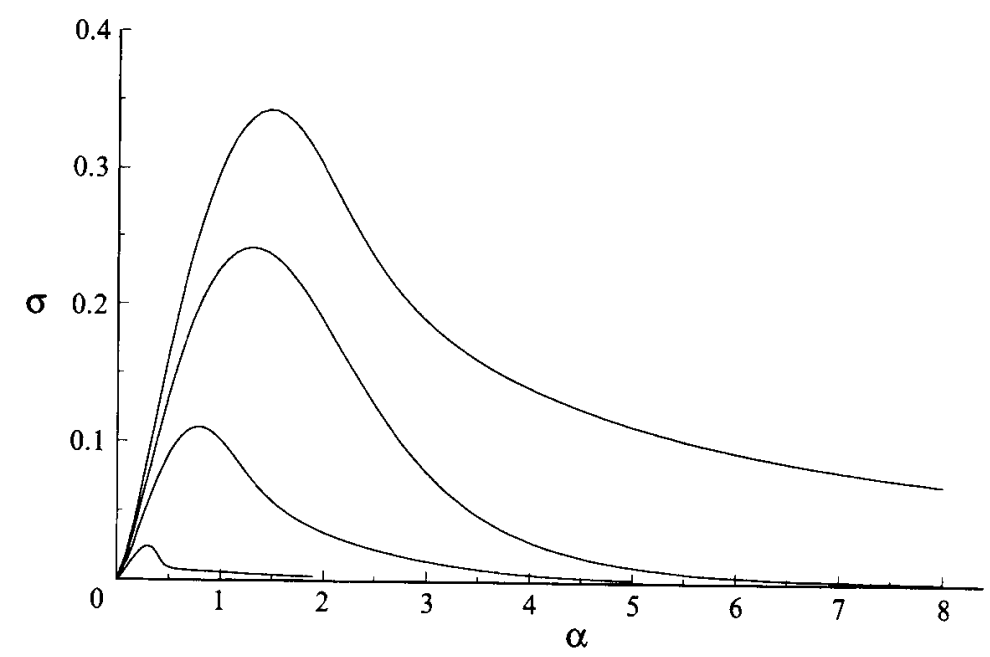

FIGURE 1. The growth rate $\sigma=\alpha c_{i}$ as a function of wavenumber $\alpha$ for the sinuous mode of a two-dimensional submerged jet with a parabolic velocity profile. The different curves are from the top for $E=0,0.05,0.1$ and 0.15 .

pressure symmetric about the centreline. We thus apply the conditions

$$
\begin{aligned}
& \eta=1, \quad p=0, \quad \text { at } y=0 \text { for the sinuous mode , } \\
& \eta=0, \quad p=1, \quad \text { at } y=0 \text { for the varicose mode . }
\end{aligned}
$$

Outside the jet in $|y|>1$ the governing equations describe a potential flow

$$
p=K \mathrm{e}^{\mp \alpha y}, \quad \eta=\mp \frac{K}{\alpha c^{2}} \mathrm{e}^{\mp \alpha y},
$$

where $K$ is a constant. This solution requires us to apply a boundary condition at the edge of the jet

$$
p=-\alpha c^{2} \eta \text { at } y=1 \text {. }
$$

The problem has therefore been reduced to integrating the elastic Rayleigh equation starting from the sinuous/varicose centreline conditions at $y=0$ and integrating to the potential flow matching condition at the edge of the jet at $y=1$. A solution will only be possible if the complex wave speed $c=c_{r}+\mathrm{i} c_{i}$ is an eigenvalue for the particular real wavenumber $\alpha$. We are interested in the growth rate $\sigma=\alpha c_{i}$ as a function of the wavenumber $\alpha$ and elasticity $E$.

This problem has been tackled numerically, using a fourth-order Runge-Kutta scheme to integrate the differential equation and a Newton-Raphson method to find the eigenvalue. The step-size for the integration was automatically reduced where the coefficients in the equation became large. Sometimes it was possible to avoid large coefficients by integrating the differential equation along a path in the complex $y$-plane.

Results for the growth rate as a function of wavenumber are shown for various values of the elasticity number $E$ in figure 1 for the sinuous mode and in figure 2 for the varicose mode. We found only one unstable eigensolution for a given wavenumber. The growth rate starts from zero for long waves as $\alpha \rightarrow 0$, increases to a maximum and then decreases to zero again as $\alpha \rightarrow \infty$. We found no finite wavenumber cut off 


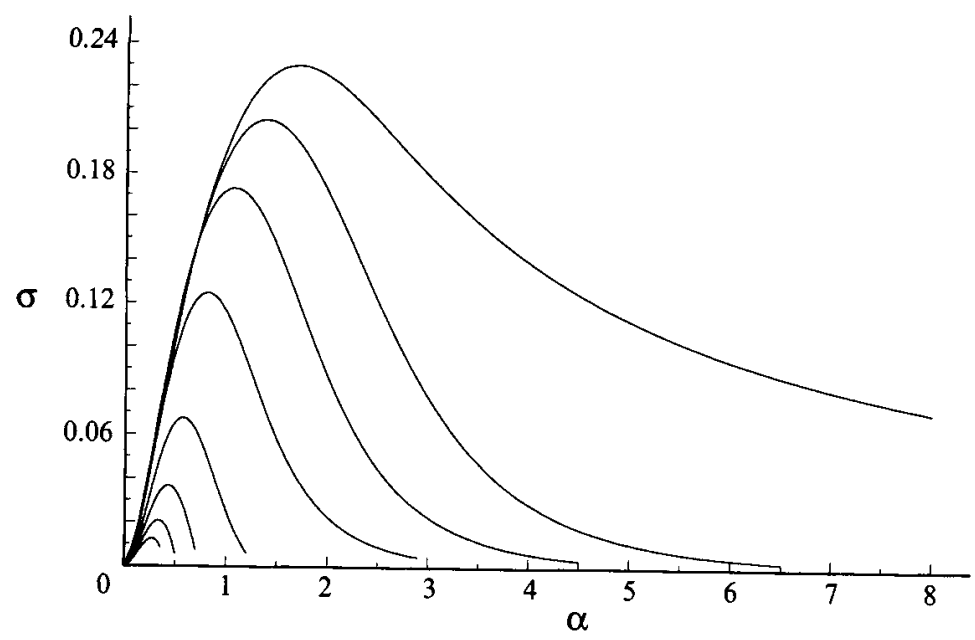

FIGURE 2. The growth rate $\sigma=\alpha c_{i}$ as a function of wavenumber $\alpha$ for the varicose mode of a two-dimensional submerged jet with a parabolic velocity profile. The different curves are from the top for $E=0,0.05,0.1,0.2,0.4,0.6,0.8$ and 1.0. Further curves for $0<E<0.05$ are shown in figure 9 .

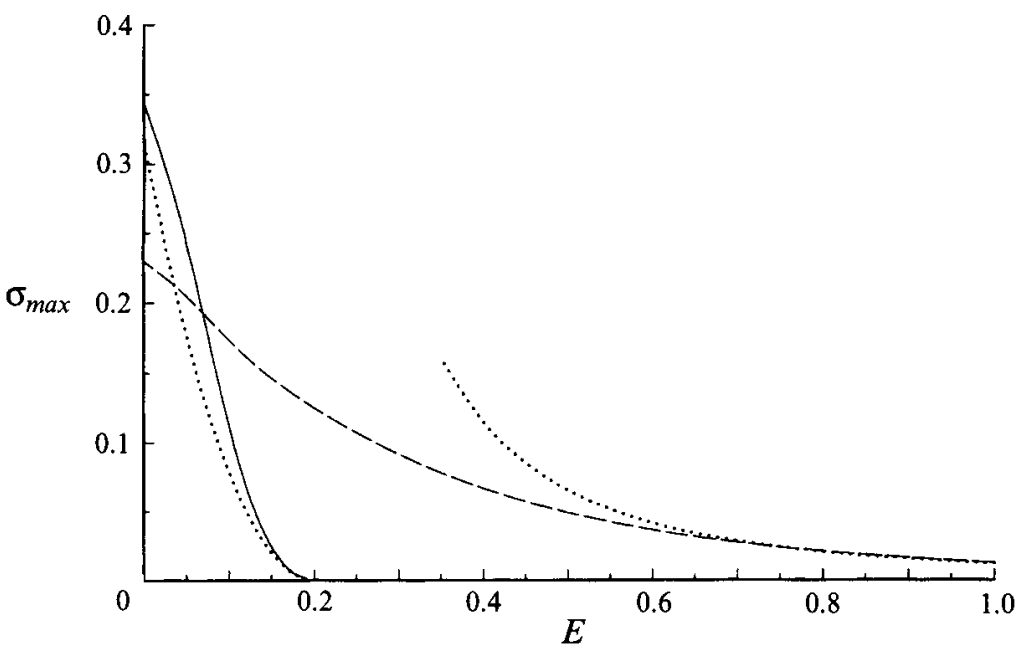

FIGURE 3. The maximum growth rate of the two-dimensional jet as a function of the elasticity for the sinuous mode (continuous curve) and the varicose mode (dashed curve). The dotted curves are the asymptotic results for long waves.

corresponding to a neutrally stable mode. The effect of the elasticity is to reduce the growth rate and to push the maximum growth rate to longer wavelengths.

Figure 3 gives the maximum growth rate as a function of the elasticity. For the inelastic jet, the sinuous mode is more unstable than the varicose mode. As the elasticity is increased the sinuous mode becomes stabilized for disturbances of all wavelengths at $E=0.2$, but the varicose mode is always unstable albeit with a reducing growth rate. The varicose mode becomes the more unstable at about $E=0.08$. 
For long wavelengths $\alpha \rightarrow 0$, it is possible to make some analytic progress. At long wavelengths, the sinuous mode has constant streamline displacements at leading order and a small variation of pressure

$$
\begin{gathered}
\eta \sim 1, \\
p \sim \alpha^{2} \int_{0}^{y}\left[\left(1-y^{2}-c\right)^{2}-8 E y^{2}\right] \mathrm{d} y .
\end{gathered}
$$

Substituting this into the boundary condition at $y=1$ yields the dispersion relation

$$
\alpha^{2}\left[\frac{8}{3}\left(\frac{1}{5}-E\right)-\frac{4}{3} c+c^{2}\right]=-\alpha c^{2} .
$$

Thus as $\alpha \rightarrow 0$

$$
c \sim \frac{2}{3} \alpha \pm i \frac{2}{3} \alpha^{1 / 2}\left[6\left(\frac{1}{5}-E\right)-\alpha\right]^{1 / 2} .
$$

This predicts a stabilization of the sinuous mode at $E=0.2$, as seen in the numerical results. It also predicts as $E \rightarrow 0.2$ a maximum growth rate at $\alpha_{\max } \sim \frac{9}{2}\left(\frac{1}{5}-E\right)$ of

$$
\sigma_{\max } \sim \frac{9 \sqrt{3}}{2}\left(\frac{1}{5}-E\right)^{2}
$$

which is plotted as a dotted line in figure 3. This analysis shows that the driving force of the instability is the centrifugal force of the fluid moving around the curved streamlines, and that this is partially or fully stabilized by the hoop stress of the curved tensioned streamlines. As $\alpha \rightarrow 0$, the wave speed $c \rightarrow 0$ so that to leading order the centrifugal force in the above expression for the pressure is $\rho U^{2}$. The first correction comes from the reduction of the velocity relative to the small wave speed in $\rho(U-c)^{2}$. More general velocity profiles $U(y)$ are similarly stabilized to long-wavelength sinuous disturbances if

$$
G>\int_{0}^{b} \rho U^{2} \mathrm{~d} y / \int_{0}^{b} A_{11} \mathrm{~d} y .
$$

The varicose mode is not fully stabilized by the elasticity. Instead the growth rate decreases and the most unstable wavelength becomes long as $E \rightarrow \infty$. At long wavelengths $\alpha \rightarrow 0$, the leading approximation to the varicose mode is a pressure constant across the jet and streamline displacements such that the acceleration along the jet balances this streamwise pressure gradient, i.e.

$$
\eta \sim \int_{0}^{y} \frac{\mathrm{d} y}{\left(1-y^{2}-c\right)^{2}-8 E y^{2}} .
$$

Our computations show that as $\alpha \rightarrow 0$ the wave speed tends to the centreline velocity of unity with a small imaginary correction. We therefore set $c=1+\mathrm{i} \delta$ and approximate the above integrand by $1 /\left(-\delta^{2}-8 E y^{2}\right)$ for $E \gg 1$. Thus the major contribution to the integral comes from a thin region of thickness $\delta /(8 E)^{1 / 2}$, and outside this region

$$
\eta=\eta_{1} \sim-\frac{\pi}{2 \delta(8 E)^{1 / 2}} .
$$

Substituting this estimate into the boundary condition on the edge of the jet $y=1$ gives a leading-order approximation to the dispersion relation. The first correction 
comes for a small correction to the pressure

$$
p \sim 1+\alpha^{2} \int_{0}^{y} \eta\left[y^{4}-8 E y^{2}\right] \mathrm{d} y \sim 1-\alpha^{2} \eta_{1} \frac{8}{3} E
$$

for $E \gg 1$. With this correction term the dispersion relation is

$$
\sigma=\frac{\alpha^{2} \pi}{4(2 E)^{1 / 2}}\left(1-\frac{8}{3} E \alpha\right) .
$$

The maximum growth rate thus occurs at $\alpha_{\max }=0.25 / E$ and is

$$
\sigma_{\max }=\frac{\pi}{192 \sqrt{2}} E^{-5 / 2},
$$

which is plotted in figure 3. The above analysis shows that the varicose mode is not fully stabilized by elasticity because the associated eigenfunction is peaked near the axis of the jet where the normal stresses vanish.

The mechanism of the varicose instability is different from that of the sinuous mode. At long wavelengths the varicose mode propagates with the centreline velocity and grows slowly. We consider this instability in the frame that moves with the centreline velocity. The fluid outside the jet is then moving backwards past a slowly growing, but stationary disturbance of the jet. Where the jet is thicker, the streamlines outside crowd together and hence the pressure drops by Bernoulli (the reduced pressure accelerating approaching fluid and retarding receding fluid). For long waves, there is little variation of the pressure across the jet and hence the pressure inside the jet is also reduced where the jet is thicker. Bernoulli then implies that the streamlines must also be crowded like those outside the jet, so long as there is flow along the streamlines, i.e. everywhere except near the centre of the jet. A consequence of this crowding of the streamlines where the jet is thicker is that the streamlines must dilate near the centre of the jet. But near the centre of the jet, which is a sort of critical layer, the flow would be stagnant if there was no growth. The low-pressure region however 'attracts' fluid (i.e. accelerates fluid towards it), which further dilates the streamlines locally and therefore makes the disturbance grow. Elasticity reduces this growth by reducing the thickness of the region where the low-pressure attracts fluid: just off the centreline, the normal stresses are non-zero and their perturbations can instead balance the low pressure by dilating the streamlines.

\section{Axisymmetric jets}

We consider a base flow of a submerged jet of width $b$ and maximum velocity $U_{0}$ and with a parabolic profile

$$
U=\left\{\begin{array}{ll}
U_{0}\left(1-r^{2} / b^{2}\right) & \text { in } r<b \\
0 & \text { otherwise }
\end{array} .\right.
$$

The associated base stress for the Oldroyd-B fluid is

$$
G A_{11}=\left\{\begin{array}{ll}
8 G \tau^{2} U_{0}^{2} r^{2} / b^{4} & \text { in } r<b \\
0 & \text { otherwise }
\end{array} .\right.
$$

We again non-dimensionalize the problem using the velocity $U_{0}$ and the length $b$, with the one non-dimensional group measuring the strength of the elasticity $E=G \tau^{2} / \rho b^{2}$. We look for a possibly non-axisymmetric perturbation with pressure $p$ and radial displacement proportional to $\cos n \theta$, and circumferential displacements proportional to $\sin n \theta$. The elastic Rayleigh equation then reduces to 


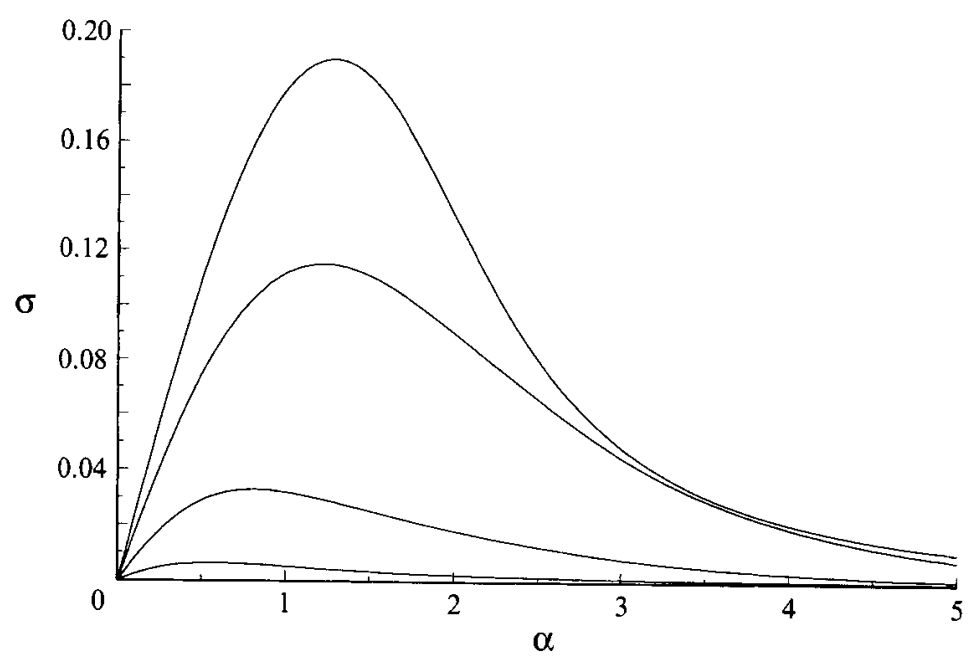

FIGURE 4. The growth rate $\sigma=\alpha c_{i}$ as a function of wavenumber $\alpha$ for the $n=1$ sinuous mode for an axisymmetric submerged jet with a parabolic velocity profile. The different curves are from the top for $E=0,0.05,0.1$ and 0.15

$$
\begin{aligned}
& \frac{\mathrm{d} q}{\mathrm{~d} r}=\left(\alpha^{2}+\frac{n^{2}}{r^{2}}\right) \frac{r}{\left(1-r^{2}-c\right)^{2}-8 E r^{2}} p, \\
& \frac{\mathrm{d} p}{\mathrm{~d} r}=\frac{\left(1-r^{2}-c\right)^{2}-8 E r^{2}}{r} q .
\end{aligned}
$$

Here $q / \alpha^{2} r$ is the radial displacement of the streamlines. Regularity as $r \rightarrow 0$ requires

$$
p \sim r^{n} \quad \text { and } q \sim n r^{n} /(1-c)^{2} .
$$

The potential flow outside the jet sets a boundary condition at $r=1$ :

$$
\frac{p}{q}=\frac{c^{2} K_{n}(\alpha)}{\alpha K_{n}^{\prime}(\alpha)}
$$

with modified Bessel function $K_{n}$. The same numerical technique as for the planar case was employed to find the growth rate $\sigma=\alpha c_{i}$ as a function of the wavenumber $\alpha$ and elasticity $E$.

Figure 4 gives the growth rate for the $n=1$ sinuous mode as a function of the wavenumber. As in the two-dimensional jet, the growth rate tends to zero at long and short wavelengths, and has a maximum at an intermediate wavenumber. Increasing the elasticity decreases the maximum growth rate, and again the most unstable wavelength becomes long. At a critical value of the elasticity $E=E *=0.3756$ the $n=1$ sinuous mode is fully stabilized (and at 0.2254 for $n=2,0.1759$ for $n=3$ and 0.1518 for $n=4)$. An examination of the numerical results for the growth rate suggests that as $E \rightarrow E$.

$$
\sigma \approx \alpha\left(E_{*}-E\right)^{3.2}\left(1-\frac{\alpha}{5\left(E_{*}-E\right)}\right) .
$$

We have no detailed analysis for this last result, but anticipate that the centrifugal mechanism is similar to that for the sinuous mode of the two-dimensional jet. The 


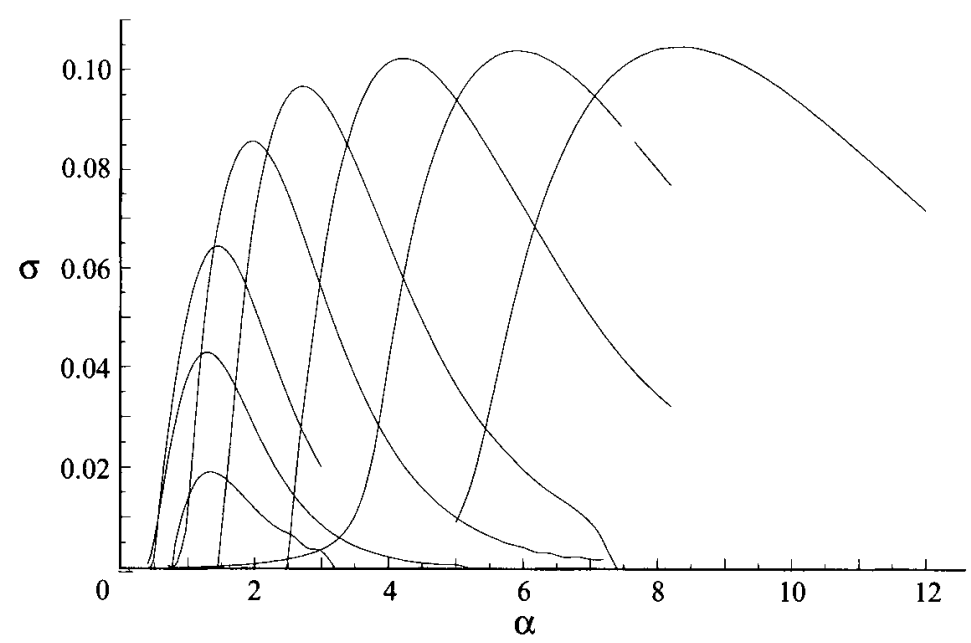

FIGURE 5. The growth rate $\sigma=\alpha c_{i}$ as a function of wavenumber $\alpha$ for the varicose mode of an axisymmetric jet. The different curves are from the right for $E=0.0025,0.005,0.01,0.025,0.05,0.1$, 0.15 and 0.2 .

scalings are not identical because in three dimensions the fluid can move around the cross-section instead of moving the long distance of a wavelength.

With zero elasticity, the axisymmetric jet with a parabolic velocity profile was found by Batchelor \& Gill (1962) to be stable to varicose $(n=0)$ perturbations. It seems that in the radial geometry the crowding of the streamlines in the outer part of the jet cannot be matched by sufficient dilatancy of the streamlines in the critical layer on the centreline. With small elasticity, however, we find that the jet is unstable. Figure 5 gives the growth rate as a function of wavenumber at various small values of the elasticity. The varicose instability exists only for small non-zero elasticity, $0<E<0.228$. As the elasticity tends to zero, the maximum growth rate tends to a constant while the unstable wavenumbers tend to infinity. Hence the limit of zero elasticity is singular. Figure 6 gives the maximum growth rate as a function of the elasticity for both the varicose and $n=1$ sinuous mode. The sinuous mode is more unstable at small elasticity $0 \leqslant E<0.07$ and larger elasticity $0.228<E<0.376$.

The mechanism of the varicose instability of the axisymmetric jet is quite different from that of the previous modes, as the wavenumber dependence shown in figure 5 hints. It involves the interaction of two elastic waves. As the elasticity tends to zero, the real part of the velocity $c_{r}$ tends to zero and not to unity as in the case of the two-dimensional jet. Thus the critical layer is near the outer edge of the jet rather than on the centreline. Near the outer edge the elastic stress $G A_{11}$ is approximately constant at a dimensionless value of $8 E$. Corresponding to this base stress in the streamlines, elastic waves propagate at a velocity $\pm(8 E)^{1 / 2}$ relative to the flow. Approximating the flow by the constant shear $2(1-r)$ near the edge of the jet, we see that a forwards travelling elastic wave on the edge of the jet $r=1$ has the same velocity as a backwards travelling elastic wave at $r=1-(8 E)^{1 / 2}$ just inside the jet if the elasticity is small $E \ll 1$. This is the real part of the velocity of the instability. The imaginary part is found to be approximately one tenth the size of the real part, and so the instability grows slowly as it propagates. The numerical results also show that the wavelength of the fastest growing wave is approximately ten times the thickness of the critical region between $r=1$ and $r=1-(8 E)^{1 / 2}$. With $\alpha_{\max } \propto 1 /(8 E)^{1 / 2}$ and 


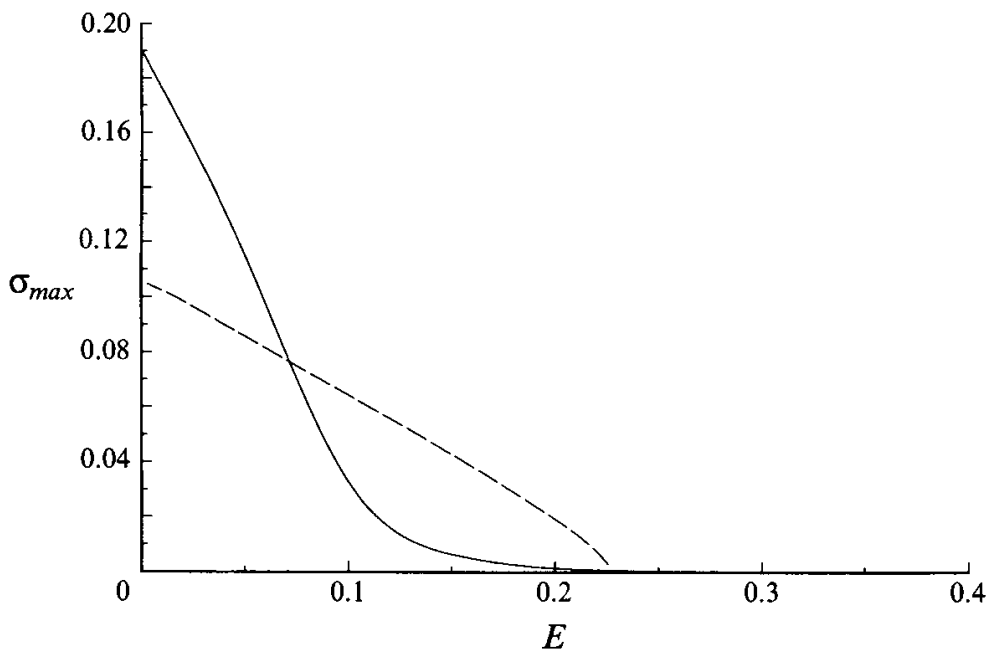

FIGURE 6. The maximum growth rate as a function of the elasticity for the sinuous mode (continuous curve) and the varicose mode (dashed curve) of the axisymmetric jet.

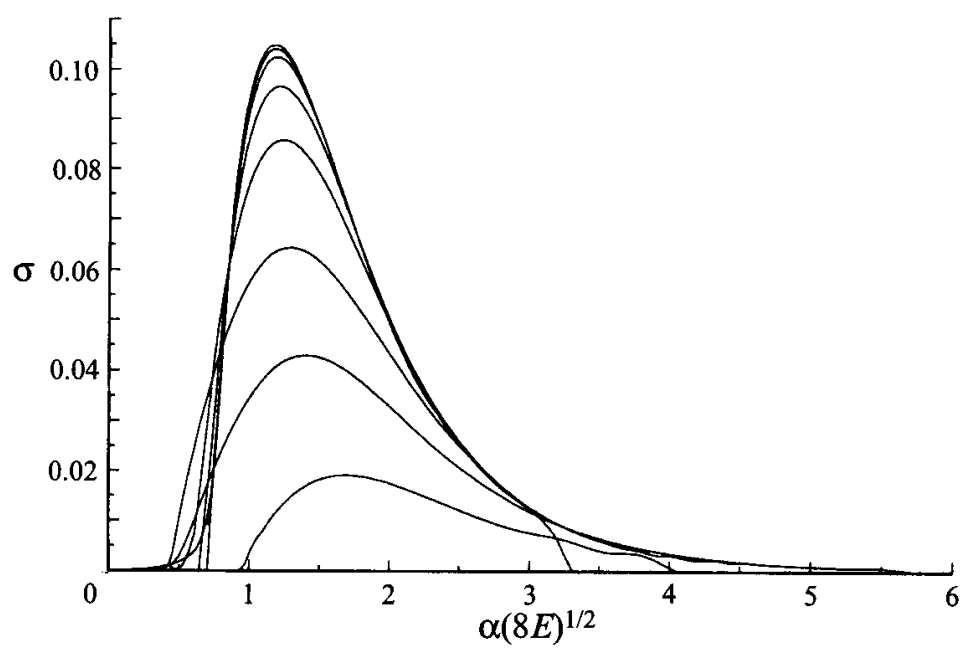

FIGURE 7. The growth rate $\sigma=\alpha c_{i}$ as a function of the rescaled wavenumber $\alpha(8 E)^{1 / 2}$ for the varicose mode of an axisymmetric jet. The different curves are from the top for $E=0.0025,0.005$, $0.01,0.025,0.05,0.1,0.15$ and 0.2 .

$c_{i} \propto(8 E)^{1 / 2}$, it follows that the maximum growth rate should be independent of the elasticity $E$ as it tends to zero. This is demonstrated by figure 7, where we have plotted the growth rate as a function of a rescaled wavenumber $\alpha \times(8 E)^{1 / 2}$. One consequence of this observation is that this instability mode is universal for all jet profiles having a discontinuity in the shear rate. One can easily write down the rescaled equations for the asymptotic form of the instability as $E \rightarrow 0$, but we have not solved these equations because no further simplification (such as the rescaled wavelength of the fastest growing wave being either small or large) is available.

In the critical region $1-(8 E)^{1 / 2} \leqslant r \leqslant 1$, the elasticity dominates inertia in the factor $\left(\rho(U-c)^{2}-G A_{11}\right)$ which occurs in the governing equation. (Ignoring the 


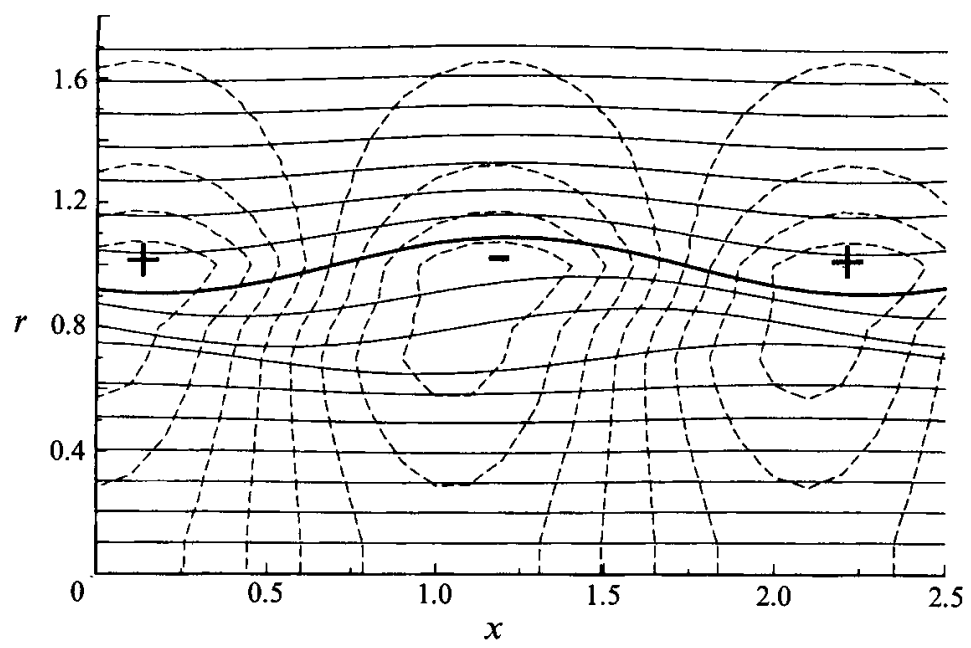

FIGURE 8. The continuous lines are the streamlines of the varicose instability for $E=0.01$ at $\alpha=3$. The boundary of the jet is indicated by the heavy line. The dashed curves are contours of pressure.

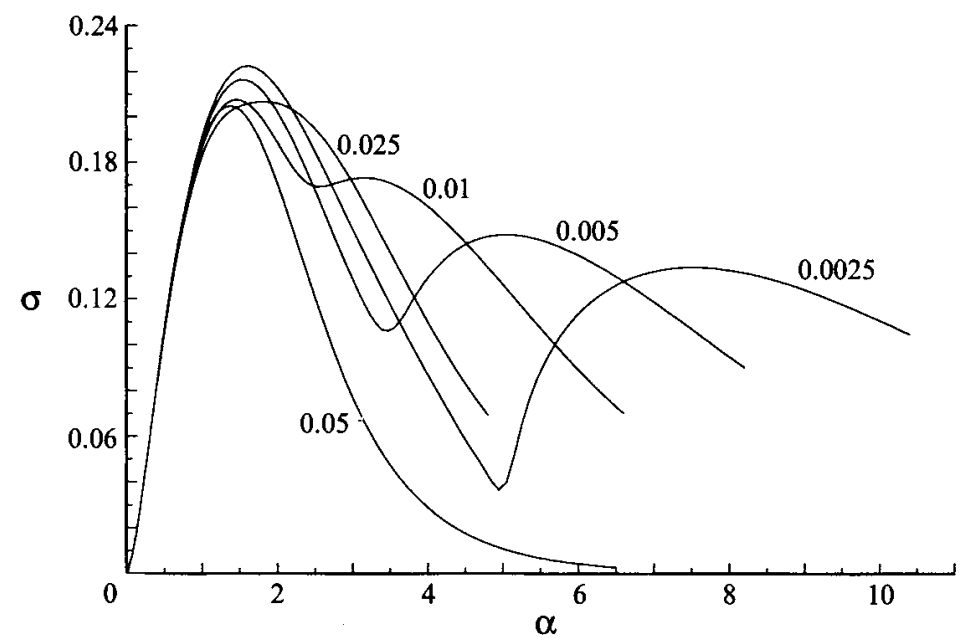

FIGURE 9. The growth rate $\sigma=\alpha c_{i}$ as a function of wavenumber $\alpha$ for the varicose mode of a two-dimensional submerged jet with a parabolic velocity profile. The different curves are labelled by the value of the elasticity $E$.

imaginary part of the wave speed, this factor vanishes at $r=1$ and $1-(8 E)^{1 / 2}$.) Just outside the jet, where there is no elasticity, inertia dominates. Hence the factor takes opposite signs on either side of the edge of the jet. The axial momemtum equation thus predicts that a low-pressure region will cause the streamlines to crowd outside the jet and to dilate just inside the jet, as shown in figure 8 . Note the streamlines are hardly deflected in the interior of the jet where the velocity and hence the inertial terms are very much larger than at the edge.

Because this $E \rightarrow 0$ instability lives near the edge of the jet, it does not know whether the jet is axisymmetric or planar nor whether the disturbance is varicose or sinuous. Thus one might have expected to see the mode in the earlier results for the 
two-dimensional jet and for the sinuous mode of the axisymmetric jet. The earlier results had, however, too large a value of the elasticity. Figure 9 gives further results for the varicose mode of the two-dimensional jet, for lower values of the elasticity. For $E<0.01$, one can see that two modes are present, one with fastest growth around $\alpha=2$ and one around $\alpha=1 /(8 E)^{1 / 2}$. The new edge mode always grows more slowly for a planar jet than the central mode. For $E>0.025$ the two modes merge.

\section{Conclusions}

Viscoelastic liquid jets in air are known to be stabilized against capillary RayleighTaylor instabilities by the presence of elastic stresses (see Goldin et al. 1969, quoted recently by Boger \& Walters 1993). We are unaware of comparable experiments with submerged jets of miscible fluids, but our calculation suggests that inertial instabilities will again be reduced by the presence of streamline tension and that the most dangerous linear sinuous mode for Newtonian fluids can be suppressed altogether by a sufficient degree of elasticity. The varicose mode of the two-dimensional parabolic jet on the other hand cannot be fully suppressed. Our results for the varicose mode of the axisymmetric jet show that elasticity may even destabilize the flow.

The computations were supported in part by the SERC CSI grant GR/H57585 and in part by the DTI LINK programme on colloids.

\section{REFERENCES}

AzAIEz, J. \& Homsy, G. M. 1994 Linear stability of free shear flow of viscoelastic liquids. J. Fluid Mech. 268, 37-69.

BAtchelor, G. K. \& Gill, A.E. 1962 Analysis of the stability of axisymmetric jets. J. Fluid Mech. 14, 529-551.

Bickley, W. G. 1937 The plane jet. Phil. Mag. 26, 268-324.

Boger, D. V. \& Walters, K. 1993 Rheological Phenomena in Focus. Elsevier.

Drazin, P. G. \& Howard, L. N. 1966 Hydrodynamic instability of parallel flow of inviscid fluids. In Advances in Applied Mechanics, vol 7 (ed. G. Kuerti), pp 1-89. Academic Press.

Goldin, M., Yerushalmi, J., Pfeffer, R. \& Shinnar, R. 1969 Breakup of a laminar capillary jet of a viscoelastic fluid. J. Fluid Mech. 38, 689-711.

Hinch, E. J., HaRris, O. J. \& Rallison, J. M. 1992 The instability mechanism for two elastic liquids being co-extruded. J. Non-Newtonian Fluid Mech. 43, 311-324.

Howard, L. N. 1961 Note on a paper by J.W. Miles. J. Fluid Mech. 10, 509-512. 